SUPPORTING MATERIAL

for

\title{
Intrinsic Base-Pair Rearrangement in the Hairpin Ribozyme Directs RNA Conformational Sampling and Tertiary Interface Formation
}

\author{
Patrick O. Ochieng, Neil A. White, Michael Feig, Charles G. Hoogstraten \\ Department of Biochemistry and Molecular Biology \\ Michigan State University, East Lansing, Michigan 48824 USA
}




\section{SUPPLEMENTARY METHODS}

The loop A RNA structures were solvated in a cubical box with TIP3P water molecules ${ }^{1,2}$ using a minimum distance of $10 \AA$ from any RNA atom to the edge of the simulation box. The systems were neutralized with $18 \mathrm{Na}^{+}$for LpA and $26 \mathrm{Na}^{+}$for the hpA construct. In the LpA simulation, excess $0.15 \mathrm{M} \mathrm{NaCl}$ (15 ion pairs) were added. The initial box dimensions were $(55.5 \AA)^{3}$ and $(62.3 \AA)^{3}$ for $\mathrm{LpA}$ and $\mathrm{hpA}$, respectively. The simulations were performed with the CHARMM36 force field ${ }^{3-7}$ Periodic boundary conditions were applied in all simulations. The particle-mesh Ewald (PME) method was used to model long-range electrostatic interactions using $(56)^{3}$ and $(54)^{3}$ cubic grids for the LpA and hpA simulations, respectively. ${ }^{8,9}$ The solutesolvent systems were initially minimized over 500 steps and then heated slowly by consecutively running 2 ps at $10 \mathrm{~K}, 4$ ps each at $20 \mathrm{~K}, 50 \mathrm{~K}, 75 \mathrm{~K}, 100 \mathrm{~K}, 125 \mathrm{~K}, 150 \mathrm{~K}, 175 \mathrm{~K}, 200 \mathrm{~K}, 220 \mathrm{~K}, 240 \mathrm{~K}$, $260 \mathrm{~K}$, and $280 \mathrm{~K}$ before running $20 \mathrm{ps}$ at at the final temperature of $298 \mathrm{~K}$. All bonds involving hydrogens were constrained holonomically by the SETTLE algorithm, allowing the use of a time step of $2 \mathrm{fs}$. The LpA production simulations were carried out in the NPT ensemble at $298 \mathrm{~K}$ and 1 bar using a Langevin thermostat with a friction coefficient of $5 \mathrm{ps}^{-1}$ and a Langevin piston with an oscillation period of $200 \mathrm{fs}$ and a decay time of $100 \mathrm{fs}$. The hpA system was simulated in the NVT ensemble at $298 \mathrm{~K}$ using a Langevin thermostat with a friction coefficient of $0.01 \mathrm{ps}^{-1}$. One simulation was carried out for LpA over $100 \mathrm{~ns}$; twenty independent simulations, each over $1 \mu \mathrm{s}$ using different random seeds to obtain different initial velocities were carried out for hpA. Individual simulations are labeled hpA1-20. The LpA simulations were run using $\mathrm{NAMD}^{10}$, version 2.7b2, whereas $\mathrm{CHARMM}^{11}$, version $\mathrm{c} 41 \mathrm{a} 2$, coupled to openMM, ${ }^{12}$ was used to take advantage of GPU acceleration for the hpA system. 
To compare force field behavior in this RNA system, we carried out parallel simulations on the LpA construct with the Amber 11 sander module using the param99 force field incorporating the bsc0 refinements for nucleic acids. ${ }^{13-15}$ In the Amber simulations, the RNA was neutralized with $18 \mathrm{Na}^{+}$counter ions using xleap at positions of high negative electric potential and solvated in a rectangular water box measuring $66.4 \AA \times 69.4 \AA \times 80.4 \AA$ with a minimum $10 \AA$ thick layer of TIP3P water molecules. $16 \mathrm{NaCl}$ pairs were added amounting to an excess salt concentration of $0.08 \mathrm{M} \mathrm{NaCl}$. Long distance electrostatic interactions were accounted for with the particle-mesh Ewald summation. ${ }^{8}$ Minimization of the solute-solvent system was done in two steps. First, the RNA was restrained with a harmonic potential force of $500 \mathrm{kcal} /\left(\mathrm{mol} \AA^{2}\right)$ in 500 steps of steepest descent minimization followed by 500 steps of conjugate gradient minimization. Second, the complete system (RNA, solvent and ions) was minimized together without restraints during 2,500 steps at a constant volume with periodic boundaries. The system was equilibrated by heating from $0 \mathrm{~K}$ to $300 \mathrm{~K}$ with 4 ps runs each at $5 \mathrm{~K}, 10 \mathrm{~K}, 15 \mathrm{~K}, 20 \mathrm{~K}, 25 \mathrm{~K}, 30 \mathrm{~K}, 35 \mathrm{~K}, 40 \mathrm{~K}$, $45 \mathrm{~K}, 50 \mathrm{~K}, 55 \mathrm{~K}, 60 \mathrm{~K}, 70 \mathrm{~K}, 75 \mathrm{~K}, 80 \mathrm{~K}, 100 \mathrm{~K}, 120 \mathrm{~K} 125 \mathrm{~K}, 140 \mathrm{~K} 150 \mathrm{~K}, 160 \mathrm{~K}, 175 \mathrm{~K}, 180 \mathrm{~K}$, 200K, 220K, 240K, 260K, 280K, 298K, and 300K in the NVT ensemble using a Langevin thermostat ${ }^{16}$ with a friction coefficient of $1 \mathrm{ps}^{-1}$ and a $10 \mathrm{kcal} /\left(\mathrm{mol} \AA^{2}\right)$ positional restraint. An additional 100 ps equilibration was then carried out at $300 \mathrm{~K}$ under NPT conditions using a Langevin thermostat and barostat without restraints followed by a production phase of $100 \mathrm{~ns}$, also in the NPT ensemble. The SHAKE algorithm ${ }^{17}$ was used to constrain covalent hydrogen bonds during the simulation, allowing a 2 fs time step. 


\section{TABLES}

Table S1. Comparison of NOE distance constraints with simulation averages using Amber and CHARMM ${ }^{\mathrm{a}}$

\begin{tabular}{lllll}
\hline Atom Pair & $\begin{array}{l}\text { NOE distance } \\
\text { constraint }(\AA)\end{array}$ & $\begin{array}{l}\text { Solution } \\
\text { structure }(\AA)\end{array}$ & $\begin{array}{l}\text { CHARMM36 } \\
\text { MD average }(\AA)\end{array}$ & $\begin{array}{l}\text { Amber param99- } \\
\text { bsc0 } \\
\text { MD average }(\AA)\end{array}$ \\
\hline U+2H1'- C+3H5 & $1.8-3.0$ & 3.0 & $2.5(0.04)$ & $5.9(1.1)$ \\
A9H1'- C+3H6 & $2.5-4.0$ & 3.9 & $5.4(0.15)$ & $8.5(0.3)$ \\
A9H1'- C+3H5 & $3.0-6.5$ & 4.4 & $6.3(0.21)$ & $9.5(0.2)$ \\
A9H1'- C+3H1' & $3.0-6.5$ & 3.2 & $4.5(0.10)$ & $5.4(0.4)$ \\
A9H4'- C+3H1' & $1.8-4.0$ & 4.0 & $7.6(0.07)$ & $7.7(0.3)$ \\
\hline
\end{tabular}

${ }^{a}$ NOE distances as reported in the loop A solution structure ${ }^{18}$ in comparison to distances in the average structures derived from $100 \mathrm{~ns}$ MD simulations with the indicated force fields. Statistical errors of the MD averages based on block averaging are given in parentheses. 
Table S2: Selected backbone and base-pair parameters of average structures derived from CHARMM36 and Amberff99-bsc0 simulations of the A-1C mutant of loop A. The numbers in bold indicate a significant conformational deviation with respect to the reported solution structure. $^{18}$

\begin{tabular}{cccc|ccc}
\hline \multicolumn{3}{c|}{ Pseudorotation phase angle (degrees) } & \multicolumn{3}{c}{$\chi$ torsion angle (degrees) } \\
\hline Residue & $\begin{array}{c}\text { Solution } \\
\text { structure }\end{array}$ & $\begin{array}{c}\text { CHARMM36 } \\
\text { MD average }\end{array}$ & $\begin{array}{c}\text { Amberff99 } \\
\text { MD } \\
\text { average }\end{array}$ & $\begin{array}{c}\text { Solution } \\
\text { structure }\end{array}$ & $\begin{array}{c}\text { CHARMM36 } \\
\text { MD average }\end{array}$ & $\begin{array}{c}\text { Amberff99 } \\
\text { MD } \\
\text { average }\end{array}$ \\
\hline A4 & 7.2 & 14.8 & 11.5 & -165.6 & -161.9 & -79.1 \\
C5 & 6.5 & 13.8 & 19.3 & -156.8 & -156.8 & -147.3 \\
A6 & 6.7 & 9.0 & 11.9 & -162.9 & -152.7 & -158.2 \\
A7 & 59.5 & 10.1 & 14.9 & -169.5 & -153.6 & -148.3 \\
G8 & 181.3 & 173.7 & $\mathbf{1 7 . 3}$ & -113.4 & -107.8 & -173.3 \\
A9 & 19.9 & 8.2 & 12.7 & -162.9 & -163.8 & 175.6 \\
A10 & 9.9 & 10.2 & 8.9 & -171.6 & -165.1 & -169.7 \\
G11 & 0.4 & 13.3 & 7.2 & -175.6 & -168.5 & -154.7 \\
G12 & 4.8 & 10.3 & 10.8 & -170.4 & -165.7 & -166.9 \\
C13 & 7.1 & 37.5 & $\mathbf{5 5 . 1}$ & -161.1 & -152.9 & -147.5 \\
G16 & 4.8 & 15.9 & 11.7 & -179.2 & -165.3 & $\mathbf{6 7 . 6}$ \\
C17 & 2.6 & 11.8 & 17.5 & -162.9 & -160.3 & -173.5 \\
C18 & 16.3 & 14.4 & 17.6 & -154.6 & -160.9 & -156.5 \\
C19 & 5.2 & 13.4 & 15.1 & -172.6 & -156.7 & -143.3 \\
G20 & 186.2 & 175.7 & 169.4 & -95.5 & -116.8 & -121.5 \\
U21 & 16.8 & 152.6 & 173.3 & -173.4 & -109.4 & -144.9 \\
C22 & 40.1 & 11.9 & 24.7 & -140.9 & -158.8 & -165.6 \\
U23 & 15.42 & 22.5 & 25.4 & -153.9 & -152.3 & -160.0 \\
G24 & 2.07 & 13.6 & 11.9 & -171.8 & -149.2 & -162.4 \\
U25 & 8.8 & ND & 13.1 & ND & ND & -154.8 \\
\hline
\end{tabular}

\begin{tabular}{cccc|ccc}
\hline & \multicolumn{3}{c|}{ Shear (degrees) } & \multicolumn{3}{c}{ Buckle (degrees) } \\
\hline Base-pair & $\begin{array}{c}\text { Tinoco's } \\
\text { structure }\end{array}$ & $\begin{array}{c}\text { CHARMM36 } \\
\text { MD average }\end{array}$ & $\begin{array}{c}\text { Amberff10 } \\
\text { MD average }\end{array}$ & $\begin{array}{c}\text { Tinoco's } \\
\text { structure }\end{array}$ & $\begin{array}{c}\text { CHARMM36 } \\
\text { MD average }\end{array}$ & $\begin{array}{c}\text { Amberff10 } \\
\text { MD average }\end{array}$ \\
\hline A4-U25 & 0.2 & -1.1 & $\mathbf{7 . 7}$ & 6.8 & 30.6 & 35.2 \\
C5-G24 & 1.2 & -0.4 & -0.1 & 12.6 & 4.8 & 4.2 \\
A6-U23 & -0.4 & -0.5 & 0.5 & 29.8 & 17.7 & $\mathbf{3 . 4 1}$ \\
A7-C22 & 4.1 & 5.4 & 6.9 & -14.1 & -7.9 & -11.4 \\
G8-U21 & -0.3 & 1.9 & -4.8 & 163.8 & $\mathbf{3 8 . 8}$ & $\mathbf{- 2 . 5}$ \\
A9-G20 & -4.9 & -5.5 & -2.6 & -19.3 & -19.2 & $-\mathbf{- 1 6 6 . 9}$ \\
A10-C19 & -2.9 & -2.8 & -2.6 & -14.6 & 0.6 & $\mathbf{- 1 6 7 . 1}$ \\
G11-C18 & -0.6 & 0.1 & -2.1 & -2.4 & 0.5 & $\mathbf{1 7 2 . 5}$ \\
G12-C17 & -0.5 & -0.4 & -2.1 & 1.9 & -3.9 & $\mathbf{1 6 8 . 8}$ \\
C13-G16 & 0.2 & 0.5 & 4.5 & 6.8 & 0.6 & $\mathbf{1 7 5 . 2}$ \\
\hline
\end{tabular}


Table S3: Most populated states from Markov state analysis.

\begin{tabular}{|c|c|c|c|c|}
\hline Index & $\begin{array}{l}\text { Population } \\
(\%)^{\mathrm{a}}\end{array}$ & $\begin{array}{l}\text { Central loop } \\
\text { base-pairing }\end{array}$ & Extra-helical bases $^{c}$ & $\begin{array}{l}\text { Average RMSD to docked } \\
\text { loop A structure }[\AA]^{\mathrm{d}}\end{array}$ \\
\hline 175 & 6.54 & AA..xxCA & & 3.61 \\
\hline 20 & 6.06 & AAga..CA & $\underline{\mathrm{G} 8}$ & 3.82 \\
\hline 0 & 5.25 & AA..cgCA & $\underline{\mathrm{A} 9}$ & 3.78 \\
\hline 123 & 5.07 & AA....UA & & 4.17 \\
\hline 170 & 5.01 & AA....CA & & 3.53 \\
\hline 143 & 4.43 & AA..cgCA & & 4.49 \\
\hline 132 & 3.49 & AAga..UA & & 4.14 \\
\hline 181 & 3.21 & GA....UA & & 4.17 \\
\hline 94 & 2.98 & AAga..CA & $\underline{\mathrm{U}+2}$ & 4.07 \\
\hline 246 & 2.10 & AAga..CA & & 4.33 \\
\hline 145 & 1.90 & AA....UA & $\mathrm{C}+3$ & 5.52 \\
\hline 155 & 1.70 & AA....UA & $\mathrm{C}+3$ & 6.16 \\
\hline 61 & 1.66 & AA..cg.. & & 3.97 \\
\hline 249 & 1.58 & AA..xxCA & & 2.78 \\
\hline 160 & 1.55 & AA....CA & & 4.82 \\
\hline 100 & 1.39 & AA..ugUA & $\underline{\mathrm{C}+3}$ & 5.92 \\
\hline 201 & 1.29 & GA....CA & & 5.84 \\
\hline 26 & 1.28 & AAga..UA & $\mathrm{C}+3$, & 4.91 \\
\hline 59 & 1.25 & AA....CA & $\underline{\mathrm{U}+2}$ & 4.30 \\
\hline 237 & 1.23 & AA....CA & & 5.24 \\
\hline 208 & 1.09 & AA....CA & & 3.48 \\
\hline 67 & 1.02 & AAgacgCA & $\underline{\mathrm{U}+2}$ & 4.38 \\
\hline 108 & 0.97 & Gaga..UA & $\underline{\mathrm{C}+3}$ & 5.07 \\
\hline
\end{tabular}




\begin{tabular}{|l|l|l|l|l|}
\hline 174 & 0.90 & AA..... & $\underline{\mathrm{G}+1, \mathrm{U}+2}$ & 5.81 \\
\hline 1 & 0.88 &.. aa..UA & $\underline{\mathrm{C}+3}$ & 5.29 \\
\hline 227 & 0.87 & AAga..UA & $\underline{\mathrm{C}+3}$ & 5.07 \\
\hline 136 & 0.82 & $\mathrm{AA} \ldots \ldots$ & & 4.03 \\
\hline 157 & 0.80 & AA....UA & $\underline{\mathrm{C}+3}$ & 5.95 \\
\hline 150 & 0.79 &.. aa..UA & $\underline{\mathrm{C}+3}$ & 5.01 \\
\hline 129 & 0.75 & GA....CA & & 6.42 \\
\hline 195 & 0.72 & AAga..CA & $\underline{\mathrm{U}+2}$ & 4.36 \\
\hline 42 & 0.72 & AA....CA & $\underline{\mathrm{U}+2}$ & 5.52 \\
\hline 5 & 0.71 & AA..ug.. & $\underline{\mathrm{G}+1, \mathrm{U}+2, \mathrm{C}+3}$ & 5.57 \\
\hline 12 & 0.71 & AA....UA & $\underline{\mathrm{A} 9, \mathrm{C}+3}$ & 5.08 \\
\hline 167 & 0.65 & AAga..UA & $\underline{\mathrm{C}+3}$ & 5.15 \\
\hline 47 & 0.65 & AA..ug.. & & 2.98 \\
\hline 231 & 0.64 & GA....UA & & 4.27 \\
\hline 118 & 0.64 & $\ldots \ldots . . \mathrm{UA}$ & & 4.99 \\
\hline 190 & 0.63 & AAgacgCA & $\underline{\mathrm{U}+2}$ & \\
\hline 128 & 0.60 & AA...cgCA & & 3.63 \\
\hline
\end{tabular}

${ }^{a}$ percentage of total snapshots assigned to state; ${ }^{b}$ see main text for notation; ${ }^{c}$ bases where N1 (guanine/adenine) or N3 (cytosine/thymine) was not within $5 \AA$ distance from any base atom of either neighboring base or the respective base-pair forming base(s) for more than $50 \%$ of the snapshots; ${ }^{\mathrm{d}}$ heavy atom RMSD to docked loop conformation from 1M5K. ${ }^{19}$ 


\section{DISCUSSION}

Tables S1 and S2 report comparisons of 100 ns simulations of a mutant loop A beginning from the reported solution structure of the molecule ${ }^{18}$ with two different force fields (see Methods). Table S1 reports comparisons of the average structures from these trajectories with five characteristic proton-proton distances for which NOE constraints are available. ${ }^{18}$ In the simulation with Amber param99-bsc0, the average simulation structure violated all but one of the listed NOE distance constraints, whereas only two NOEs were violated with CHARMM36 (A9 $\mathrm{H} 1^{\prime}-\mathrm{C}+3 \mathrm{H} 6$ and $\mathrm{A} 9 \mathrm{H} 1^{\prime}-\mathrm{C}+3 \mathrm{H} 1^{\prime}$ ), and the former distance in the CHARMM36 simulation was closer to the NOE range than with the Amber force field. We note that the reported solution structure displays NOE distances at the upper limit of the constraint range for both contacts violated in the CHARMM simulations, suggesting that these distance constraints may themselves be mutually inconsistent in the original dataset. In addition, we analyzed ribose sugar puckering and base geometries for the calculated structures and again found better agreement with the experimental data for the average structure generated by the CHARMM36 force field compared to the Amber99-bsc0 average structure (Table S2). Based on these initial simulation results in our particular system, the CHARMM36 force field was chosen for production simulations of the wild-type loop A constructs. We emphasize that this single computation should not be taken as a systematic comparison of these two force fields for nucleic acids. Indeed, such more systematic

studies as have been done (see, for example, recent careful work from the Cheatham lab ${ }^{20}$ ) have pointed up deficiencies of various types in most or all current nucleic acid force fields, emphasizing the importance of further developments in the area. 


\section{REFERENCES}

1. Jorgensen, W. L. Quantum and Statistical Mechanical Studies of Liquids .10.

Transferable Intermolecular Potential Functions for Water, Alcohols, and Ethers Application to Liquid Water. J. Am. Chem. Soc. 1981, 103, 335-340.

2. Jorgensen, W. L.; Chandrasekhar, J.; Madura, J. D.; Impey, R. W.; Klein, M. L.

Comparison of Simple Potential Functions for Simulating Liquid Water. J. Chem. Phys. 1983, 79, 926-935.

3. Foloppe, N.; MacKerell, A. D. All-Atom Empirical Force Field for Nucleic Acids: I. Parameter Optimization Based on Small Molecule and Condensed Phase Macromolecular Target Data. J. Comput. Chem. 2000, 21, 86-104.

4. MacKerell, A. D.; Banavali, N. K. All-Atom Empirical Force Field for Nucleic Acids: II. Application to Molecular Dynamics Simulations of DNA and RNA in Solution. $J$. Comput. Chem. 2000, 21, 105-120.

5. Best, R. B.; Zhu, X.; Shim, J.; Lopes, P. E.; Mittal, J.; Feig, M.; Mackerell, A. D., Jr. Optimization of the Additive CHARMM All-Atom Protein Force Field Targeting Improved Sampling of the Backbone Phi, Psi and Side-Chain Chi(1) and Chi(2) Dihedral Angles. J. Chem. Theory. Comput. 2012, 8, 3257-3273.

6. MacKerell, A. D.; Bashford, D.; Bellott, M.; Dunbrack, R. L.; Evanseck, J. D.; Field, M. J.; Fischer, S.; Gao, J.; Guo, H.; Ha, S.; Joseph-McCarthy, D.; Kuchnir, L.; Kuczera, K.; Lau, F. T.; Mattos, C.; Michnick, S.; Ngo, T.; Nguyen, D. T.; Prodhom, B.; Reiher, W. E.; Roux, B.; Schlenkrich, M.; Smith, J. C.; Stote, R.; Straub, J.; Watanabe, M.; Wiorkiewicz-Kuczera, J.; Yin, D.; Karplus, M. AllAtom Empirical Potential for Molecular Modeling and Dynamics Studies of Proteins. J. Phys. Chem. B 1998, 102, 3586-3616.

7. Huang, J.; Mackerell, A. D., Jr. CHARMM36 All-Atom Additive Protein Force Field: Validation Based on Comparison to NMR Data. J. Comput. Chem. 2013, 34, 2135-2145.

8. Darden, T.; York, D.; Pedersen, L. Particle Mesh Ewald - An N.Log(N) Method for Ewald Sums in Large Systems. J. Chem. Phys. 1993, 98, 10089-10092.

9. Essmann, U.; Perera, L.; Berkowitz, M. L.; Darden, T.; Lee, H.; Pedersen, L. G. A Smooth Particle Mesh Ewald Method. J. Chem. Phys. 1995, 103, 8577-8593.

10. Phillips, J. C.; Braun, R.; Wang, W.; Gumbart, J.; Tajkhorshid, E.; Villa, E.; Chipot, C.; Skeel, R. D.; Kale, L.; Schulten, K. Scalable Molecular Dynamics With NAMD. J. Comput. Chem. 2005, 26, 1781-1802. 
11. Brooks, B. R.; Brooks, C. L., III; Mackerell, A. D., Jr.; Nilsson, L.; Petrella, R. J.; Roux, B.; Won, Y.; Archontis, G.; Bartels, C.; Boresch, S.; Caflisch, A.; Caves, L.; Cui, Q.; Dinner, A. R.; Feig, M.; Fischer, S.; Gao, J.; Hodoscek, M.; Im, W.; Kuczera, K.; Lazaridis, T.; Ma, J.; Ovchinnikov, V.; Paci, E.; Pastor, R. W.; Post, C. B.; Pu, J. Z.; Schaefer, M.; Tidor, B.; Venable, R. M.; Woodcock, H. L.; Wu, X.; Yang, W.; York, D. M.; Karplus, M. CHARMM: the Biomolecular Simulation Program. J. Comput. Chem. 2009, 30, 1545-1614.

12. Eastman, P.; Friedrichs, M. S.; Chodera, J. D.; Radmer, R. J.; Bruns, C. M.; Ku, J. P.; Beauchamp, K. A.; Lane, T. J.; Wang, L. P.; Shukla, D.; Tye, T.; Houston, M.; Stich, T.; Klein, C.; Shirts, M. R.; Pande, V. S. OpenMM 4: A Reusable, Extensible, Hardware Independent Library for High Performance Molecular Simulation. J. Chem. Theory Comput. 2013, 9, 461-469.

13. Cornell, W. D.; Cieplak, P.; Bayly, C. I.; Gould, I. R.; Merz, K. M.; Ferguson, D. M.; Spellmeyer, D. C.; Fox, T.; Caldwell, J. W.; Kollman, P. A. A Second Generation Force Field for the Simulation of Proteins, Nucleic Acids, and Organic Molecules (Vol 117, Pg 5179, 1995). J. Am. Chem. Soc. 1996, 118, 2309.

14. Cheatham, T. E.; Cieplak, P.; Kollman, P. A. A Modified Version of the Cornell Et Al. Force Field With Improved Sugar Pucker Phases and Helical Repeat. Journal of Biomolecular Structure \& Dynamics 1999, 16, 845-862.

15. Perez, A.; Marchan, I.; Svozil, D.; Sponer, J.; Cheatham, T. E., III; Laughton, C. A.; Orozco, M. Refinement of the AMBER Force Field for Nucleic Acids: Improving the Description of Alpha/Gamma Conformers. Biophys. J. 2007, 92, 3817-3829.

16. Adelman, S. A.; Doll, J. D. Generalized Langevin Equation Approach for Atom-SolidSurface Scattering - General Formulation for Classical Scattering Off Harmonic Solids. J. Chem. Phys. 1976, 64, 2375-2388.

17. Ryckaert, J. P.; Ciccotti, G.; Berendsen, H. J. C. Numerical-Integration of Cartesian Equations of Motion of A System With Constraints - Molecular-Dynamics of NAlkanes. Journal of Computational Physics 1977, 23, 327-341.

18. Cai, Z.; Tinoco, I., Jr. Solution Structure of Loop A From the Hairpin Ribozyme From Tobacco Ringspot Virus Satellite. Biochemistry 1996, 35, 6026-6036.

19. Rupert, P. B.; Ferre-D'Amare, A. R. Crystal Structure of a Hairpin Ribozyme-Inhibitor Complex With Implications for Catalysis. Nature 2001, 410, 780-786.

20. Bergonzo, C.; Henriksen, N. M.; Roe, D. R.; Cheatham, T. E., III Highly Sampled Tetranucleotide and Tetraloop Motifs Enable Evaluation of Common RNA Force Fields. RNA. 2015, 21, 1578-1590. 